\title{
Reduced white matter integrity as a neural correlate of social cognition deficits in schizophrenia.
}

\section{$\operatorname{AUTHOR}(S)$ :}

Miyata, Jun; Yamada, Makiko; Namiki, Chihiro; Hirao, Kazuyuki; Saze, Teruyasu; Fujiwara, Hironobu; Shimizu, Mitsuaki; ... Sawamoto, Nobukatsu; Hayashi, Takuji; Murai, Toshiya

\section{CITATION:}

Miyata, Jun ... [et al]. Reduced white matter integrity as a neural correlate of social cognition deficits in schizophrenia.. Schizophrenia research 2010, 119(1-3): 232-239

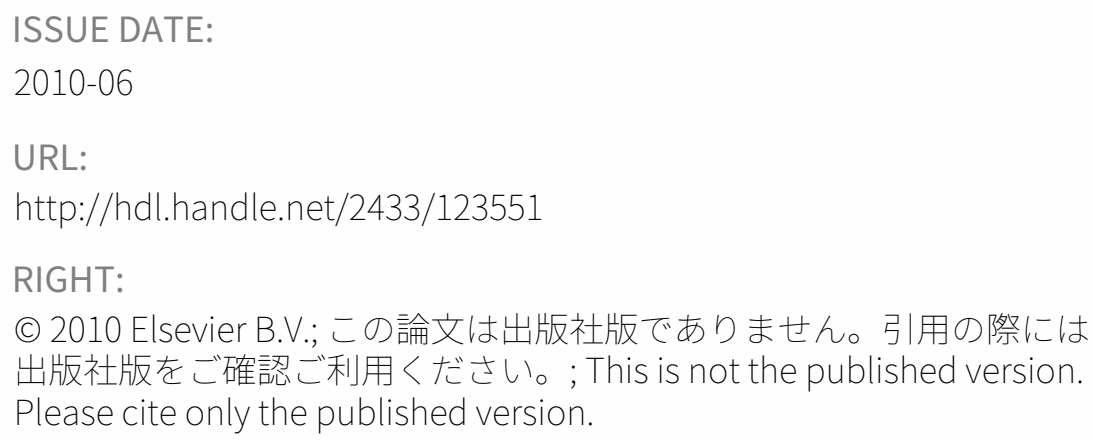

C 2010 Elsevier B.V.; この論文は出版社版でありません。引用の際には 出版社版をご確認ご利用ください。; This is not the published version. Please cite only the published version. 


\section{Reduced white matter integrity as a neural correlate of social cognition deficits in schizophrenia}

Jun Miyata ${ }^{a)}$, Makiko Yamada $^{\text {a) }}$, Chihiro Namiki ${ }^{\text {a), b), Kazuyuki Hirao }}{ }^{\text {a), }}$ Teruyasu Saze $^{\text {a) }}$, Hironobu Fujiwara ${ }^{\text {a) }}$, Mitsuaki Shimizu ${ }^{a)}$, Ryosaku Kawada ${ }^{a}$, Hidenao Fukuyama ${ }^{\text {b) }}$, Nobukatsu Sawamoto ${ }^{\text {b) }}$, Takuji Hayashi ${ }^{\text {a) }}$, Toshiya Murai $^{\text {a) }}$

${ }^{a}$ Department of Neuropsychiatry, Graduate School of Medicine, Kyoto University, 54 Shogoin-Kawaharacho, Kyoto 606-8507, Japan

${ }^{b}$ Human Brain Research Center, Graduate School of Medicine, Kyoto University, 54 Shogoin-Kawaharacho, Kyoto 606-8507, Japan

\section{CORRESPONDENCE TO:}

Jun Miyata, M.D.

Department of Neuropsychiatry, Graduate School of Medicine, Kyoto University

54 Shogoin-Kawaharacho, Kyoto 606-8507, Japan

Tel: +81-75-751-3386, Fax: +81-75-751-3246

E-mail: miyata10@kuhp.kyoto-u.ac.jp 


\begin{abstract}
Background: The pathology of schizophrenia is thought to involve multiple gray and white matter regions. A number of studies have revealed impaired social cognition in schizophrenia. Some evidence suggests an association of this social cognition deficit with gray matter reductions in 'social brain' areas. However, no study has yet revealed the association between social cognition abilities and white matter abnormalities in schizophrenia patients.
\end{abstract}

Methods: Twenty-six schizophrenia patients and 27 healthy controls underwent the Perception of Affect Task (PAT), which consisted of four subtasks measuring different aspects of emotion attribution. Voxelwise group comparison of white matter fractional anisotropy (FA) was performed using tract-based spatial statistics (TBSS). The relation between impaired social cognition ability and FA reduction was examined in patients for each subtask, using simple regression analysis within brain areas that showed a significant FA reduction in patients compared with controls. The same correlational analysis was also performed for healthy controls in the whole brain.

Results: Schizophrenia patients showed reduced emotion attribution ability compared with controls in all four subtasks. The facial emotion perception subtask showed a significant correlation with FA reductions in the left occipital white matter region and left posterior callosal region. The correlational analyses in healthy controls revealed no significant correlation of FA with any of the PAT subtasks.

Conclusions: Our voxelwise correlational analysis of white matter provided a potential neural basis for the social cognition impairments in schizophrenia, in support of the 
disconnection hypothesis.

Key Words: Corpus callosum, diffusion tensor imaging, disconnection hypothesis, facial emotion perception, inferior longitudinal fasciculus, tract-based spatial statistics. 


\section{Introduction}

Magnetic resonance imaging (MRI) studies on patients with schizophrenia have consistently reported gray matter volume reductions in frontal and temporal cortical regions, medial temporal lobe structures, the basal ganglia, and the thalamus (Ellison-Wright et al., 2008; Shenton et al., 2001). This disorder is thought to arise as a result of disrupted connectivity between these gray matter regions (Friston, 1998). Diffusion tensor imaging (DTI) provides information about white matter tracts and their organization based on water diffusion. DTI enables the detection of subtle white matter abnormalities, and a reduction in fractional anisotropy (FA) implies decreased white matter tract integrity. A number of studies have demonstrated FA reductions in white matter in diverse areas including frontal white matter, the corpus callosum (CC), and the cingulum, in schizophrenia patients (Kubicki et al., 2007; Walterfang et al., 2006). These findings suggest that white matter abnormalities may be an anatomical substrate for the 'disconnection hypothesis’ (Friston, 1998) of schizophrenia.

On the other hand, recent neuroimaging and lesion studies have revealed a network of gray matter regions underlying social cognition. The amygdala and the orbitofrontal cortex have been found to make important contributions to facial emotion expression processing (Adolphs et al., 1994; Hornak et al., 1996): the medial and orbitofrontal cortices play key roles in mentalizing ability, often referred to as the theory of mind (ToM; Baron-Cohen et al., 1994; Fletcher et al., 1995); the temporal pole and the superior temporal sulcus make important contributions to both of these abilities (Brunet-Gouet and Decety, 2006); and the inferior parietal lobe has a critical role in the judgment of agency (Chaminade and Decety, 
2002). In regard to schizophrenia, a number of studies of schizophrenia patients have revealed impaired social cognition abilities, and an abnormal hemodynamic response in the brain regions mentioned above during social cognition tasks (Brune, 2005; Brunet-Gouet and Decety, 2006; Mandal et al., 1998). In addition to these functional abnormalities, some studies have revealed associations between impaired social cognition ability and structural abnormalities in gray matter regions critical for social cognition (Fujiwara et al., 2007; Hirao et al., 2008; Yamada et al., 2007).

Considering the complexity of cognitive functions such as social cognition, which requires the coordinated functioning of a widely distributed network of gray matter regions, it is possible that social cognition impairments may be caused by disrupted connectivity between gray matter regions. To date, however, the association between impaired social cognition and white matter abnormality has been little examined in schizophrenia patients. To the best of our knowledge, an earlier study by our research group (Fujiwara et al., 2007) was the first to investigate this potential association. This study, which used a region of interest (ROI) method, did not reveal an association between impaired emotion attribution performance and FA reduction in the anterior cingulum bundle in schizophrenia.

To fully investigate such an association, a correlational analysis in the whole brain is necessary. In the present study, we employed the Perception of Affect Task (PAT; Rau, 1993), a test battery that encompasses a broad range of social cognitive factors, such as perspective taking and empathy. To perform voxelwise correlational analysis, we employed a recently developed technique called tract-based spatial statistics (TBSS) (Smith et al., 2006). TBSS maps each subject's DTI data onto a common white matter tract center 
('skeleton'), and is robust to registration confounds. We hypothesized that impaired emotion attribution task performance in patients would have a positive correlation with FA reduction in white matter that connects gray matter regions relevant for social cognition. To explore if the individual difference in social cognition task performance among the normal population has neural correlates in white matter, we also performed a correlational analysis of healthy controls.

\section{Materials and methods}

\subsection{Subjects}

Twenty-six schizophrenia patients, diagnosed based on the patient edition of the Structured Clinical Interview for DSM-IV Axis I Disorders (SCID), were studied. None of the patients had comorbid psychiatric disorders. All patients were receiving antipsychotic medication. The Positive and Negative Syndrome Scale (PANSS) (Kay et al., 1987) was used to assess the severity of clinical symptoms. Twenty-seven healthy controls, matched with the patient group in age, sex, handedness and education levels were recruited. The controls had no history of psychiatric illness, as determined by the non-patient edition of the SCID, and there was no history of psychotic disorders among their first-degree relatives. Exclusion criteria for all individuals included a history of head trauma, neurological illness, serious medical or surgical illness, and substance abuse. These patients and healthy controls partially overlapped with participants in our earlier studies (Fujiwara et al., 2007; Miyata et al., 2009). All participants were physically healthy at the time of scanning and psychological tests. After receiving a complete description of the study, all participants 
gave written informed consent. The study design was approved by the Committee on Medical Ethics of Kyoto University.

\subsection{Psychological tests}

To confirm the participants' basic visuoperceptual ability for facial stimuli, a short version of the Benton Facial Recognition Test (BFRT) (Benton et al., 1983) was used. Participants were asked to match the faces of identical individuals from six choices, which were shown in varying views and light conditions.

For the assessment of the participants' social cognition ability, we administered the PAT battery, which examines the ability to attribute emotions to facial expressions and to protagonists in complex social situations. The PAT battery comprises four subtasks designed to separately assess verbal, visual, and verbal-visual processing abilities.

Subtask 1 involved matching of verbally described social situations with verbal emotion labels. Short stories describing emotional situations were presented. From a list of seven emotion labels (happiness, sadness, fear, anger, disgust, surprise, and neutral), participants were asked to choose the one that best matched the feeling of the main protagonist in each situation.

Subtask 2 involved matching of emotional facial stimuli with verbal emotion labels. Participants were provided with the same emotion label list as subtask 1, and were requested to choose the most appropriate labels for presented emotional facial stimuli, selected from the Picture of Facial Affect series (Ekman and Friesen, 1976).

Subtask 3 involved matching of verbally described social situations with emotional faces. 
Participants were presented with the same short stories as in subtask 1 . They were provided with a list of seven facial expressions of an individual from the Picture of Facial Affect series and were asked to choose the facial expression most appropriately matching the feeling of the main protagonist.

Subtask 4 involved matching of emotional faces with non-verbal social situations. Seven photographs of social situations were provided. A human figure representing one of the seven emotions was indicated by an arrow in each photograph. The faces of these figures were not visible. The same facial stimuli used in subtask 2 were presented, and participants were asked to choose the most appropriate human figure.

Each subtask consisted of 35 stimuli, five items for each of seven emotions. These subtasks were performed in this fixed order for all participants.

\subsection{MRI acquisition}

DTI data were acquired using single-shot spin-echo echo-planar sequences, on a 3.0-T MRI unit (Trio; Siemens, Erlangen, Germany) with a 40-mT/m gradient and a receiver-only eight-channel phased-array head coil. A generalized autocalibrating partially parallel acquisition algorithm was applied for parallel imaging using a reduction factor of two, 24 additional autocalibrating phase-encoding steps in the center of k-space, and a 75\% partial Fourier technique in the phase-encoding direction. The parameters were as follows: echo time 79 ms, repetition time 5200 ms, $128 \times 128$ matrix, field of view $220 \times 220$ mm, 40 contiguous axial slices of $3.0 \mathrm{~mm}$ thickness. Motion-probing gradients were applied along 12 non-collinear directions with a $b$ factor of $700 \mathrm{~s} / \mathrm{mm}^{2}$ after one non diffusion-weighted 
image $\left(b=0 \mathrm{~s} / \mathrm{mm}^{2}\right)$ was obtained. Scanning was repeated four times to enhance the signal-to-noise ratio.

\subsection{Data processing and statistical analysis}

\subsubsection{Psychological data analysis}

Statistical analyses for BFRT and PAT data were performed in SPSS version 12.0 (SPSS Inc., Chicago). BFRT scores of both groups were compared using two-tailed t-tests. Group comparisons of performance in each of the four PAT subtasks were performed using $2 \times 7$ two-way analyses of variance (ANOVAs), with group as a between-subjects factor and emotion category as a within-subjects factor. For analyses assessing repeated measures effects, violations of sphericity assumptions were accounted for by correcting the degrees of freedom according to Huynh-Feldt (Vasey and Thayer, 1987). The statistical significance level was set at $\mathrm{P}<0.05$.

\subsubsection{DTI data processing and group comparison}

DTI data processing was performed using FSL ver. 4.1 (http://www.fmrib.ox.ac.uk/fsl). All DTI source data were corrected for eddy currents and head motion by registering all data to the first $b=0$ image of the first repetition, with affine transformation. Averaged data from these four repetitions were created for each subject, and FA maps were calculated using the FDT program. For voxelwise statistical analysis, TBSS ver. 1.2, which is part of FSL, was used. Briefly, all subjects' FA data were aligned into a common space using the nonlinear registration tool FNIRT, which uses a b-spline representation of the registration 
warp field (Rueckert et al., 1999). All transformed FA images were averaged to create a mean FA image, which was then thinned to create a mean FA skeleton, taking only the centers of white matter tracts common to all the subjects. Voxel values of each subject's FA map were projected onto the skeleton by searching the local maxima along the perpendicular direction from the skeleton.

Voxelwise permutation-based nonparametric inference (Nichols and Holmes, 2001) was performed on skeletonized FA data, using FSL Randomize ver 2.1. We first performed group comparisons, using an analysis of covariance (ANCOVA) design, with age and gender as nuisance covariates. Both covariates were centered (demeaned) before being fed into the design matrix, and pre-removed from the data before implementing the permutation tests. Both control-patient and patient-control contrasts were tested, with 10000 permutations. Multiple comparisons were corrected using the recently developed technique of threshold-free cluster enhancement (TFCE) (Smith and Nichols, 2009). TFCE avoids making an arbitrary choice of the cluster-forming threshold, while preserving the sensitivity benefits of clusterwise correction. From a raw statistical image, TFCE produces an output image in which the voxel value represents a weighted sum of the local clustered signal. Voxelwise statistical inference was made on these output images and the significance level was set at $\mathrm{p}<0.01$, corrected for family-wise error rate. The resultant significant FA reduction/increase areas in patients were used as inclusion masks in the following voxelwise correlational analysis.

\subsubsection{Correlational analysis}


Voxelwise simple regression analyses of schizophrenia patients were performed using TBSS. Design matrices were created for each PAT subtask, modeling total subtask scores as explanatory variables. The above-mentioned masks were used to include only those voxels with significant group differences. The permutation-based nonparametric inference was undertaken with 10000 permutations. The statistical significance level was thresholded for correction of multiple comparisons using a false discovery rate (FDR) (Genovese et al., 2002) of 0.05 . The fiber tracts corresponding to the clusters were identified with reference to the Johns Hopkins University DTI-based White Matter Atlas (http://cmrm.med.jhmi.edu) (Mori et al., 2005) and Wakana et al. (2004).

To explore if there is any correlation between social cognitive ability and white matter integrity in the normal population, the same correlational analyses were performed on healthy controls for the whole skeleton. The threshold for statistical significance was set at $\mathrm{p}<0.01$, corrected for multiple comparisons by TFCE, owing to the exploratory nature of this analysis.

\subsubsection{Analysis of medication effect}

We then analyzed the impacts of medication type and dosage on our results. First, we divided patients according to their medication type (typical or atypical antipsychotics or both, and each atypical antipsychotic separately), and compared demographic, clinical, and psychological data, and the mean FA values of each region showing a significant correlation with PAT subtasks in the above-mentioned analyses. Calculation of mean FA was done using the command-line utilities of FSL (FSLUTILS). Analyses of variance 
(ANOVAs) or chi-square tests were performed for analyses of parametric or non-parametric variables, respectively. The threshold for statistical significance was set at $p<0.05$. Second, we explored the association between FA and medication dosage using simple regression analyses for the whole skeleton. Both positive and negative correlations were tested, and the threshold for statistical significance was a set at TFCE corrected $\mathrm{p}<0.01$.

\section{Results}

\subsection{Demographic and clinical data}

The demographic and clinical data are shown in Table 1. The patient group consisted mainly of chronic patients with relatively mild symptom severity. The majority of patients were taking atypical antipsychotics. Among the 16 patients who were taking atypical antipsychotics only, seven were taking riperidone, four were taking olanzapine, one was taking quetiapine, and four were taking more than one atypical antipsychotic. Among the last group, two were taking perospirone in combination with risperidone or olanzapine. No patient was taking clozapine.

\subsection{Psychological data}

Table 1 shows the results of the BFRT and PAT. There was no significant difference between the two groups in terms of BFRT performance.

The repeated measures ANOVAs for PAT performance revealed significant main effects of group in all four subtasks: $\mathrm{F}(1,51)=8.02, \mathrm{P}=0.007$ in subtask $1 ; \mathrm{F}(1,51)=12.51, \mathrm{P}=0.001$ in subtask 2; $\mathrm{F}(1.51)=16.00, \mathrm{P}<0.001$ in subtask 3 ; and $\mathrm{F}(1,51)=14.00, \mathrm{P}<0.001$ in subtask 
4, with schizophrenia patients performing worse than healthy controls. There were significant main effects of emotion category in all subtasks: $F(6,306)=14.25, \varepsilon_{\mathrm{HF}}=0.88$, $\mathrm{P}<0.001$ in subtask $1 ; \mathrm{F}(6,306)=55.88, \varepsilon_{\mathrm{HF}}=0.73, \mathrm{P}<0.001$ in subtask $2 ; \mathrm{F}(6,306)=27.89$, $\mathrm{P}<0.001$ in subtask 3; and $\mathrm{F}(6,306)=14.06, \varepsilon_{\mathrm{HF}}=0.94, \mathrm{P}<0.001$ in subtask 4 . There was, however, no significant group by emotion interaction in any of the subtasks.

\subsection{Imaging data}

Schizophrenia patients showed a cluster of significant FA reductions compared with controls. This cluster included bilateral deep white matter in the frontal, temporal and occipital lobes, a large portion of the CC, and the corona radiata (Figure 1). Because there was no significant cluster of FA increase in patients, the following correlational analysis in patients was performed only within the FA reduction mask.

Patients' performance in subtask 2 showed a strong positive correlation with two white matter regions (Table 2 and Figures 2 and 3). One of these regions was located in the left occipital white matter. This region consisted of the callosal interhemispheric fibers, the inferior longitudinal fasciculus (ILF), the inferior occipitofrontal fasciculus (IOFF), the posterior thalamic radiation (PTR), and the corticopontine tract (CPT). The other region was located in the left posterior CC, which was identified as containing callosal interhemispheric fibers as well as fibers from the posterior cingulate cortex (PCC). Correlation coefficients at the peak voxels in these regions were 0.77 and 0.76 , respectively. The other three subtasks showed no significant correlations with FA reduction.

On the other hand, the correlational analyses in healthy controls revealed no significant 
correlation of FA with any of the PAT subtasks.

\subsection{Medication effect}

ANOVAs revealed no significant difference among patients taking typical or atypical antipsychotics or both, in any of the demographic, clinical, or psychological data, or mean FA values for the left occipital or parietal regions. There was only one patient taking quetiapine alone, and no patient took perospirone alone. Therefore, we compared the data among the risperidone alone group, the olanzapine alone group, and those patients taking more than one atypical antipsychotic, for comparison of atypical antipsychotic types. The ANOVAs revealed no significant group difference in any of the demographic, clinical, or psychological data, or mean FA values.

The correlational analyses between FA and dosage revealed no positive or negative correlation.

\section{Discussion}

This is, to the best of our knowledge, the first study to demonstrate an association between reduced white matter integrity and social cognition deficits in schizophrenia. Voxelwise correlational analysis in schizophrenia patients revealed a positive correlation between two white matter areas of FA reduction and performance on an emotion attribution task. These results indicate that, in addition to the previously demonstrated regional gray matter deficits, structural disconnectivity among gray matter networks may also contribute to social cognition deficits in schizophrenia. This finding extends our insight into the neural 
basis of social cognition impairment in schizophrenia patients.

The current study replicates the behavioral findings of our earlier study (Fujiwara et al., 2007): patients performed worse than healthy controls in all PAT subtasks. No interaction between diagnosis and emotion category was found, indicating that the deficit was of emotion in general, rather than being specific to any category of emotion.

The result of TBSS group comparison revealed a relatively large cluster of reduced FA in schizophrenia patients. Similar patterns of FA reduction have been robustly reported in earlier voxel-based DTI studies (Kyriakopoulos et al., 2008a; Mori et al., 2007; Shergill et al., 2007). The mask also contained the regions reported in our previous TBSS study (Miyata et al., 2009), which used a stricter statistical threshold.

In the correlational analyses of patients, PAT subtask 2, a facial emotion recognition task, showed a strong association with two white matter regions: one in the left occipital white matter region, and another in the left posterior CC region. The latter was comprised mainly of callosal interhemispheric fibers, and also contained fibers from the PCC. By contrast, the former was located in a merging area of three families of fibers: projection fibers (PTR and CPT), hemispheric association fibers (ILF and IOFF), and callosal interhemispheric fibers. Because multiple fiber tracts are contained in both regions, caution must be taken in interpretation. This is especially true for the occipital region. However, recent studies indicate a critical role of callosal interhemispheric fibers and the ILF in facial emotion recognition. Tamietto et al. (2006), using a tachistoscopic paradigm, demonstrated interhemispheric cooperation in facial emotion perception in healthy subjects, for both negative and positive emotions. Emotion processing impairments have also been 
documented in patients with agenesis of the CC (Paul et al., 2007). In addition, interhemispheric connectivity disturbances have been suggested to play a major role in the pathophysiology of schizophrenia (Crow, 1998), and support the category-nonspecific impairment of facial emotion processing in schizophrenia patients (Mandal et al., 1998). Our study provides, for the first time, anatomical evidence supporting the involvement of disturbed callosal interhemispheric connectivity in the impaired facial emotion recognition in schizophrenia patients.

On the other hand, although the precise function of the ILF is less clear, recent studies have suggested that this tract is involved in semantic processing (Vigneau et al., 2006) and visual memory (Catani et al,, 2003). In particular, the ILF is suggested to play a role in processing the emotional valence of visual stimuli. It is considered to mediate the fast transmission of visual stimuli to anterior temporal regions and neuromodulatory back-projections from the amygdala to early visual processing areas (Catani et al., 2003). It has been predicted previously that deficits in this fiber tract would result in impaired facial emotion recognition in schizophrenia patients (Ashtari et al., 2007; Phillips et al., 2009). In addition, a very recent longitudinal study (Karlsgodt et al., 2009) revealed that lower FA in the ILF at baseline was predictive of deterioration in social and role functioning at follow-up in subjects at ultra-high risk of psychosis; indeed, functional outcome has been shown to be critically affected by facial emotion perception (Couture et al., 2006). Thus, our study is the first to provide at least partial support for the contribution of ILF to the facial emotion recognition deficit in schizophrenia patients.

Performance in three other subtasks, on the other hand, failed to show any significant 
association with FA reduction. By contrast, our earlier studies found associations between performance on these subtasks and the volume of the ACC and medial prefrontal cortex (subtasks 3 and 4), and the morphology of the ACC (subtask 1) (Fujiwara et al., 2007; Yamada et al., 2007). In these three subtasks, participants must attribute emotion to protagonists in verbal or non-verbal social scenarios; in other words, these subtasks involve so called emotional ToM or empathy. Taking these points into account, the simplest interpretation of our current results is that, unlike the facial emotion perception deficits discussed above, the impairment of emotional ToM or empathy in schizophrenia is embodied by gray matter abnormalities, not by white matter dysfunction, as indicated in our earlier study (Fujiwara et al., 2007). This may indicate that the impairment of each component or module of social cognition in schizophrenia is caused by different types of neural abnormalities: one component is affected by white matter abnormalities, another is affected by gray matter abnormalities, and yet another is affected by both of them. We should be cautious, however, when generalizing such a differential association. Another interpretation is that some white matter regions of possible association with empathy (such as the uncinate fasciculus, which connects frontal and temporal gray matter regions) were not included in our mask owing to the subtleness of the abnormality (Phillips et al., 2009). This possibility may be less likely; we also explored the correlations in the whole skeleton in patients, and found no additional significant regions outside the FA reduction mask for any subtasks, even with the liberal threshold of TFCE corrected $\mathrm{p}<0.01$. Yet another possibility is that, if the abnormalities associated with social cognition are located in white matter regions adjacent to cortical gray matter, and not in the deep white matter tracts, it is 
possible that those regions were excluded from the skeleton in exchange for the reduction of misregistration in TBSS. To address the issues raised above, and to clarify which fiber tracts are associated with emotion attribution ability, a tractography study examining each fiber tract separately and an ROI-based study to examine subcortical white matter are necessary.

Several studies have revealed correlations between certain cognitive functions and FA in healthy samples (Konrad et al., 2009; Schmithorst et al., 2005). Thus, it is reasonable to hypothesize that individual differences in a social cognitive task performance are correlated with the individual variabilities of FA values in healthy people. In our study, correlational analyses in healthy controls revealed no association between FA values and any of the PAT subtasks. However, this should be cautiously interpreted, as all the subtasks showed low variances in healthy controls (Table 1), which may lead to negative findings in the correlational analyses. Use of a test battery that can better differentiate social cognition abilities among the normal population is necessary. Another possibility is in the smaller variance of FA values in healthy people than in schizophrenia patients; we checked this possibility by calculating mean FA within the whole skeleton for each subject, and found that the control group had a smaller variance of this whole skeleton mean FA (S.D. of the whole skeleton mean FA: 0.0117 in healthy people, 0.0184 in patients. Levene’s test, $\mathrm{P}=0.034)$. This indicated that we needed a larger sample size to detect any correlation in a healthy population; indeed, both Konrad et al. (2009) and Schmithorst et al. (2005) had larger sample sizes of healthy participants compared to that in our study. Taking the above into consideration, it is possible that the association between the individual difference of a 
social cognitive ability and the individual variability of white matter integrity is subtler relative to the association between the impairment of that ability and the abnormality of the white matter microstructure.

There was no effect of medication type on any of the demographic, clinical, psychological, or FA data. Nor was there any association between FA and medication dosage. Thus, our results are considered to be independent of medication effects, although we should keep in mind the following limitations. First, in the medication type comparison, some categories contain only a small number of patients, which could reduce the statistical power. Second, although our negative findings regarding a correlation between FA and medication dosage are consistent with the findings of most early studies (Kyriakopoulos et al., 2008b), several studies have shown significant positive or negative correlations (Kuroki et al., 2006; Minami et al., 2003; Okugawa et al., 2004). To address the effect of medication, a study with a larger sample size, which can analyze the association between FA and dosage for each medication type, would be ideal.

In addition to the methodological limitations raised above, several points should be mentioned. First, not a few patients $(\mathrm{N}=10)$ were taking more than one antipsychotic, and this may have obscured the effect of medication on our results, especially the impact of each atypical antipsychotic. Second, we recruited mainly chronic patients with mild symptom severity. Studies on subjects with more severe symptoms or at earlier stages of disease would be desired to further elaborate our findings. Third, certain social cognition abilities may differ between males and females (Baron-Cohen et al., 2001). Our sample size was not large enough to perform correlational analysis for each gender separately. A study 
with a larger sample size would be needed to clarify the effect of gender.

In conclusion, our study demonstrates the first direct associations between regional FA reduction in two white matter areas and impaired social cognition ability in schizophrenia. Voxelwise correlational analysis of white matter extends our insight into the neural basis of the facial emotion recognition deficits in schizophrenia patients, in the light of the disconnection hypothesis. 


\section{References}

Adolphs, R., Tranel, D., Damasio, H., Damasio, A., 1994. Impaired recognition of emotion in facial expressions following bilateral damage to the human amygdala. Nature 372 669-672.

Ashtari, M., Cottone, J., Ardekani, B.A., Cervellione, K., Szeszko, P.R., Wu, J., Chen, S., Kumra, S., 2007. Disruption of white matter integrity in the inferior longitudinal fasciculus in adolescents with schizophrenia as revealed by fiber tractography. Arch Gen Psychiatry 64 1270-1280.

Baron-Cohen, S., Ring, H., Moriarty, J., Schmitz, B., Costa, D., Ell, P., 1994. Recognition of mental state terms. Clinical findings in children with autism and a functional neuroimaging study of normal adults. Br J Psychiatry 165 640-649.

Baron-Cohen, S., Wheelwright, S., Hill, J., Raste, Y., Plumb, I., 2001. The "Reading the Mind in the Eyes" Test revised version: a study with normal adults, and adults with Asperger syndrome or high-functioning autism. J Child Psychol Psychiatry 42 $241-251$.

Benton, A.L., Hamsher, K.S., Varney, N.R., Spreen, O., 1983. Facial Recognition: Stimulus and Multiple Choice Pictures. Oxford University Press, New York.

Brune, M., 2005. “Theory of mind” in schizophrenia: A review of the literature. Schizophr Bull. 31 21-42.

Brunet-Gouet, E., Decety, J., 2006. Social brain dysfunctions in schizophrenia: A review of neuroimaging studies. Psychiatry Research: Neuroimaging 148 75-92.

Catani, M., Jones, D.K., Donato, R., Ffytche, D.H., 2003. Occipito-temporal connections in 
the human brain. Brain 126 2093-2107.

Chaminade, T., Decety, J., 2002. Leader or follower? Involvement of the inferior parietal lobule in agency. NeuroReport 13 1975-1978.

Couture, S.M., Penn, D.L., Roberts, D.L., 2006. The Functional Significance of Social Cognition in Schizophrenia: A Review. Schizophr Bull. 32 S44-63.

Crow, T.J., 1998. Schizophrenia as a transcallosal misconnection syndrome. Schizophr Res. 30 111-114.

Ekman, P., Friesen, W., 1976. Pictures of Facial Affect. Consulting Psychologists Press, Palo Alto, CA.

Ellison-Wright, I., Glahn, D.C., Laird, A.R., Thelen, S.M., Bullmore, E., 2008. The anatomy of first-episode and chronic schizophrenia: An anatomical likelihood estimation meta-analysis. Am J Psychiatry 165 1015-1023.

Fletcher, P.C., Happe, F., Frith, U., Baker, S.C., Dolan, R.J., Frackowiak, R.S., Frith, C.D., 1995. Other minds in the brain: A functional imaging study of "theory of mind" in story comprehension. Cognition 57 109-128.

Friston, K.J., 1998. The disconnection hypothesis. Schizophr Res. 30 115-125.

Fujiwara, H., Hirao, K., Namiki, C., Yamada, M., Shimizu, M., Fukuyama, H., Hayashi, T., Murai, T., 2007. Anterior cingulate pathology and social cognition in schizophrenia: A study of gray matter, white matter and sulcal morphometry. Neuroimage 36 1236-1245.

Genovese, C.R., Lazar, N.A., Nichols, T., 2002. Thresholding of statistical maps in functional neuroimaging using the false discovery rate. Neuroimage 15 870-878. 
Hirao, K., Miyata, J., Fujiwara, H., Yamada, M., Namiki, C., Shimizu, M., Sawamoto, N., Fukuyama, H., Hayashi, T., Murai, T., 2008. Theory of mind and frontal lobe pathology in schizophrenia: A voxel-based morphometry study. Schizophr Res. 105 $165-174$.

Hornak, J., Rolls, E.T., Wade, D., 1996. Face and voice expression identification in patients with emotional and behavioural changes following ventral frontal lobe damage. Neuropsychologia 34 247-261.

Inagaki, A., Inada, T., 2006. Translation table of psychotropic drugs. Keio University, Tokyo.

Karlsgodt, K.H., Niendam, T.A., Bearden, C.E., Cannon, T.D., 2009. White Matter Integrity and Prediction of Social and Role Functioning in Subjects at Ultra-High Risk for Psychosis. Biol Psychiatry 66 562-569.

Kay, S.R., Fiszbein, A., Opler, L.A., 1987. The Positive and Negative Syndrome Scale (PANSS) for schizophrenia. Schizophr Bull. 13 261-276.

Konrad, A., Vucurevic, G., Musso, F., Stoeter, P., Winterer, G. 2009. Correlation of brain white matter diffusion anisotropy and mean diffusivity with reaction time in an oddball task. Neuropsychobiology 60 55-66.

Kubicki, M., McCarley, R., Westin, C.F., Park, H.J., Maier, S., Kikinis, R., Jolesz, F.A., Shenton, M.E., 2007. A review of diffusion tensor imaging studies in schizophrenia. J Psychiatr Res. 41 15-30.

Kuroki, N., Kubicki, M., Nestor, P.G., Salisbury, D.F., Park, H., Levitt, J.J., Woolston, S., Frumin, M., Niznikiewicz, M., Westin, C., Maier, S.E., McCarley, R.W., Shenton, 
M.E., 2006. Fornix integrity and hippocampal volume in male schizophrenic patients. Biol Psychiatry 60 22-31.

Kyriakopoulos, M., Vyas, N.S., Barker, G.J., Chitnis, X.A., Frangou, S., 2008a. A diffusion tensor imaging study of white matter in early-onset schizophrenia. Biol Psychiatry 63 519-523.

Kyriakopoulos, M., Bargiotas, T., Barker, G.J., Frangou, S. 2008b. Diffusion tensor imaging in schizophrenia. European Psychiatry 23 255-273.

Lehman, A.F., Lieberman, J.A., Dixon, L.B., McGlashan, T.H., Miller, A.L., Perkins, D.O., Kreyenbuhl, J., 2004. Practice guideline for the treatment of patients with schizophrenia, second edition. Am J Psychiatry 161 1-56.

Mandal, M.K., Pandey, R., Prasad, A.B., 1998. Facial expressions of emotions and schizophrenia: a review. Schizophr Bull. 24 399-412.

Minami, T., Nobuhara, K., Okugawa, G., Takase, K., Yoshida, T., Sawada, S., Ha-Kawa, S., Ikeda, K., Kinoshita, T., 2003. Diffusion tensor magnetic resonance imaging of disruption of regional white matter in schizophrenia. Neuropsychobiology 47 $141-145$

Miyata, J., Hirao, K., Namiki, C., Fujiwara, H., Shimizu, M., Fukuyama, H., Sawamoto, N., Hayashi, T., Murai, T., 2009. Reduced white matter integrity correlated with cortico-subcortical gray matter deficits in schizophrenia. Schizophr Res. 111 78-85.

Mori, S., Wakana, S., Nagae-Poetscher, L., van Zijl, P.C.M., 2005. MRI Atlas of Human White Matter. Elsevier, Amsterdam.

Mori, T., Ohnishi, T., Hashimoto, R., Nemoto, K., Moriguchi, Y., Noguchi, H., Nakabayashi, 
T., Hori, H., Harada, S., Saitoh, O., Matsuda, H., Kunugi, H., 2007. Progressive changes of white matter integrity in schizophrenia revealed by diffusion tensor imaging. Psychiatry Res. 154 133-145.

Nichols, T.E., Holmes, A.P., 2001. Nonparametric permutation tests for functional neuroimaging: a primer with examples. Hum Brain Mapp. 15 1-25.

Okugawa, G., Nobuhara, K., Minami, T., Tamagaki, C., Takase, K., Sugimoto, T., Sawada, S., Kinoshita, T. 2004. Subtle disruption of the middle cerebellar peduncles in patients with schizophrenia. Neuropsychobiology 50 119-123.

Paul, L.K., Brown, W.S., Adolphs, R., Tyszka, J.M., Richards, L.J., Mukherjee, P., Sherr, E.H., 2007. Agenesis of the corpus callosum: genetic, developmental and functional aspects of connectivity. Nat Rev Neurosci. 8 287-299.

Phillips, O.R., Nuechterlein, K.H., Clark, K.A., Hamilton, L.S., Asarnow, R.F., Hageman, N.S., Toga, A.W., Narr, K.L., 2009. Fiber tractography reveals disruption of temporal lobe white matter tracts in schizophrenia. Schizophr Res. 107 30-38.

Rau, J.C., 1993. Perception of verbal and nonverbal affective stimuli in complex partial seizure disorder [abstract]. Dissertation Abstracts Int B 54 506B.

Rueckert, D., Sonoda, L., Hayes, C., Hill, D., Leach, M., Hawkes, D., 1999. Nonrigid registration using free-form deformations: application to breast MR images. IEEE Trans Med Imag. 18 712-721.

Schmithorst, V. J., Wilke, M., Dardzinski, B. J., \& Holland, S. K. 2005. Cognitive functions correlate with white matter architecture in a normal pediatric population: a diffusion tensor MRI study. Human Brain Mapping 26 139-147. 
Shenton, M.E., Dickey, C.C., Frumin, M., McCarley, R.W., 2001. A review of MRI findings in schizophrenia. Schizophr Res. 49 1-52.

Shergill, S.S., Kanaan, R.A., Chitnis, X.A., O'Daly, O., Jones, D.K., Frangou, S., Williams, S.C., Howard, R.J., Barker, G.J., Murray, R.M., McGuire, P., 2007. A diffusion tensor imaging study of fasciculi in schizophrenia. Am J Psychiatry. 64 467-473.

Smith, S.M., Jenkinson, M., Johansen-Berg, H., Rueckert, D., Nichols, T.E., Mackay, C.E., Watkins, K.E., Ciccarelli, O., Cader, M.Z., Matthews, P.M., Behrens, T.E., 2006. Tract-based spatial statistics: voxelwise analysis of multi-subject diffusion data. Neuroimage. 31 1487-1505.

Smith, S.M., Nichols, T.E., 2009. Threshold-free cluster enhancement: Addressing problems of smoothing, threshold dependence and localisation in cluster inference. Neuroimage. 44 83-98.

Tamietto, M., Latini Corazzini, L., de Gelder, B., Geminiani, G., 2006. Functional asymmetry and interhemispheric cooperation in the perception of emotions from facial expressions. Exp Brain Res. 171 389-404.

Vasey, M.W., Thayer, J.F., 1987. The continuing problem of false positives in repeated measures ANOVA in psychophysiology: A multivariate solution. Psychophysiology. 24 479-486.

Vigneau, M., Beaucousin, V., Hervé, P.Y., Duffau, H., Crivello, F., Houdé, O., Mazoyer, B., Tzourio-Mazoyer, N., 2006. Meta-analyzing left hemisphere language areas: phonology, semantics, and sentence processing. Neuroimage. 30 1414-1432.

Wakana, S., Jiang, H., Nagae-Poetscher, L.M., van Zijl, P.C., Mori, S., 2004. Fiber 
tract-based atlas of human white matter anatomy. Radiology. 230 77-87.

Walterfang, M., Wood, S.J., Velakoulis, D., Pantelis, C., 2006. Neuropathological, neurogenetic and neuroimaging evidence for white matter pathology in schizophrenia. Neurosci Biobehav Rev. 30 918-948.

Yamada, M., Hirao, K., Namiki, C., Hanakawa, T., Fukuyama, H., Hayashi, T., Murai, T., 2007. Social cognition and frontal lobe pathology in schizophrenia: a voxel-based morphometric study. Neuroimage. 35 292-298. 


\section{Figure legends.}

\section{Figure 1.}

Regions of significant FA reduction in schizophrenia patients $(\mathrm{N}=26)$ relative to healthy controls $(\mathrm{N}=27)$. To aid visualization, regions showing significant FA reductions $(\mathrm{p}<0.01$, corrected by TFCE) are thickened using the tbss_fill script implemented in FSL (red-yellow). Results are shown overlaid on the mean FA map and the FA skeleton (green). Left-right orientation is according to the radiological convention. Axial slices from $\mathrm{z}=-6$ to 45 in MNI coordinate are shown. 


\section{Figure 2.}

Significant correlation between subtask 2 performance and FA in schizophrenia patients. To aid visualization, regions showing significant FA reductions $(\mathrm{p}<0.05$, corrected by FDR) are thickened using the tbss_fill script implemented in FSL (red-yellow). The left occipital white matter region is shown overlaid on the mean FA map and the FA skeleton (green). Panel A: axial slice, B: sagittal slice, C: coronal slice. 


\section{Figure 3.}

Significant correlation between subtask 2 performance and FA in schizophrenia patients. To aid visualization, regions showing significant FA reductions $(\mathrm{p}<0.05$, corrected by FDR) are thickened using the tbss_fill script implemented in FSL (red-yellow). The left posterior corpus callosal region is shown overlaid on the mean FA map and the FA skeleton (green). Panel A: axial slice, B: sagittal slice, C: coronal slice. 


\section{Table 1.}

Demographic, psychological, and clinical characteristics of participants

\begin{tabular}{|c|c|c|c|c|c|}
\hline & \multicolumn{2}{|c|}{ Schizophrenia $(\mathrm{n}=26)$} & \multicolumn{2}{|c|}{ Control $(n=27)$} & \multirow{2}{*}{$\begin{array}{c}\text { Statistics } \\
\mathrm{P}\end{array}$} \\
\hline & mean & S.D. & mean & S.D. & \\
\hline Age (years) & 38.4 & 9.1 & 38.2 & 8.2 & N.S. \\
\hline Sex (male/ female) & \multicolumn{2}{|c|}{$13 / 13$} & \multicolumn{2}{|c|}{$11 / 16$} & N.S. ${ }^{d}$ \\
\hline Handedness (right/ left) & \multicolumn{2}{|c|}{$25 / 1$} & \multicolumn{2}{|c|}{$26 / 1$} & N.S. ${ }^{d}$ \\
\hline Education (years) & 14.1 & 2.6 & 13.5 & 2.4 & N.S. ${ }^{c}$ \\
\hline Benton Facial Recognition Task & 22.4 & 2.8 & 23.4 & 2.0 & N.S. ${ }^{\mathrm{c}}$ \\
\hline PAT subtask 1 total score & 26.3 & 5.1 & 29.6 & 3.0 & $0.007^{*}$ \\
\hline subtask 2 total score & 26.0 & 4.2 & 29.4 & 2.7 & $0.001^{*}$ \\
\hline subtask 3 total score & 22.5 & 5.3 & 27.7 & 4.1 & $<0.001^{*}$ \\
\hline subtask 4 total score & 22.6 & 6.4 & 28.4 & 4.9 & $<0.001^{*}$ \\
\hline Age of onset (years) & 25.4 & 6.0 & - & - & - \\
\hline Duration of illness (years) & 13.5 & 9.5 & - & - & - \\
\hline Medication (mg/day, HPD equivalent) $^{\mathrm{a}}$ & 11.9 & 8.5 & - & - & - \\
\hline Atypical only / Typical only / Both ${ }^{\mathrm{b}}$ & \multicolumn{2}{|c|}{$16 / 4 / 6$} & - & - & - \\
\hline \multicolumn{6}{|l|}{ Type of atypical antipsychotic } \\
\hline Risperidone & \multicolumn{2}{|c|}{7} & & & \\
\hline Olanzapine & \multicolumn{2}{|c|}{4} & & & \\
\hline Quetiapine & \multicolumn{2}{|c|}{1} & & & \\
\hline
\end{tabular}


More than 1 atypical antipsychotic

PANSS total score

positive scale

negative scale

general psychopathology scale
4

$67.8 \quad 18.9$

16.5

6.4

17.7

6.7

33.6

10.0

${ }^{\mathrm{a}}$ Haloperidol equivalents were calculated according to the practice guidelines for the treatment of patients with schizophrenia (Inagaki et al., 2006; Lehman et al., 2004).

${ }^{\mathrm{b}}$ Atypical $=$ patients who were taking atypical antipsychotics. Typical $=$ patients taking typical antipsychotics. Both = patients taking both typical and atypical antipsychotics.

${ }^{\mathrm{c}}$ Two-tailed t tests.

${ }^{\mathrm{d}}$ Two-tailed chi-square tests.

* Main effects of group in repeated measures analyses of variance. 


\section{Table 2.}

Significant correlations between performance on the PAT subtask 2 and FA in patients.

\begin{tabular}{|c|c|c|c|c|c|c|}
\hline \multirow[b]{2}{*}{ Anatomical region } & \multirow[b]{2}{*}{$\mathrm{Z}$} & \multirow[b]{2}{*}{ Correlation coefficient $r$} & \multicolumn{3}{|c|}{ MNI coordinate } & \multirow[b]{2}{*}{ Cluster size } \\
\hline & & & $\mathrm{X}$ & $\bar{Y}$ & $\mathrm{Z}$ & \\
\hline Left occipital white matter & 4.57 & 0.77 & -27 & -62 & 16 & 9 \\
\hline Left posterior CC & 4.52 & 0.76 & -14 & -33 & 27 & 6 \\
\hline
\end{tabular}

Coordinates for the peak voxels are displayed. CC = corpus callosum. 


\section{Role of Funding Source}

This work was supported by a grant-in-aid for scientific research from the Japan Society for the Promotion of Science and the Ministry of Education, Culture, Sports, Science and Technology, Japan [20691401 to Toshiya Murai]; a grant from the Ministry of Health, Labor and Welfare, Japan [20E-3 to Toshiya Murai]; and a research grant from the Research Group for Schizophrenia sponsored by Astellas Pharma Inc. These agencies had no further role in the study design, the collection, analysis and interpretation of data, the writing of the report, or in the decision to submit the paper for publication. 


\section{Conflict of Interest}

All authors declare that they have no conflicts of interest. 


\section{Acknowledgments}

The authors wish to extend their gratitude to Miho Yoshizumi for her assistance in data acquisition and processing, and, most of all, to the patients and volunteers for participating in the study. 